depression (Simon et al, 2004). The main reason for this disparity is the lack of resources in poor countries. We therefore propose that a type of brain stimulation transcranial direct current stimulation (tDCS) - may be a satisfactory alternative to increase access to adequate antidepressant treatment.

Electroconvulsive therapy (ECT) and transcranial magnetic stimulation (TMS) are examples of brain stimulation therapy that are effective in treating depression. However, these treatments are expensive and might be associated with adverse effects (Hasey, 2001). In recent years, a simple technique of brain stimulation that seemed long forgotten has received renewed attention - tDCS. This treatment is inexpensive, easy to administer, non-invasive and painless (Nitsche et al, 2003). There are few past reports of $\mathrm{tDCS}$ in treating depression (Lolas, 1977). However, at the time of those trials much less was known about the methodological aspects and physiological effects of $\mathrm{tDCS}$ and the results were quite variable.

Preliminary, unpublished data from a randomised, sham-stimulation controlled and double-blind trial evaluating the effects of anodal stimulation of the left dorsolateral prefrontal cortex in people with depression suggest that $\mathrm{tDCS}$ is an effective treatment for major depression (further details available from the authors on request).

Thus, we have come to believe that $\mathrm{tDCS}$ might be a reasonable alternative treatment for depression in low- and middle-income countries. The device to deliver tDCS is simple, can cost less than US $\$ 10000$ and can be manufactured locally. The equipment is fully reusable and utilises one standard battery that can last several weeks. Furthermore, this treatment is easy to administer, and can be applied by technicians following appropriate instruction and training. Although further studies evaluating this method are warranted, tDCS might help to improve mental health in areas with poor resources.

Hasey, G. (200I) Transcranial magnetic stimulation in the treatment of mood disorder: a review and comparison with electroconvulsive therapy. Canadian Journal of Psychiatry, 46, 720-727.

Kuruppuarachchi, K. A. L. A. \& Wijeratne, L. T. (2004) Depression intervention in resource-poor regions (letter). British Journal of Psychiatry, 185 438-439.

Lolas, F. (1977) Brain polarization: behavioral and therapeutic effects. Biological Psychiatry, 12, 37-47.
Nitsche, M. A., Liebetanz, D., Antal, A., et al (2003) Modulation of cortical excitability by weak direct current stimulation - technical, safety and functiona aspects. Supplements to Clinical Neurophysiology, 56, 255-276.

Simon, G. E., Fleck, M., Lucas, R., et al (2004) Prevalence and predictors of depression treatment in an international primary care study. American Journal of Psychiatry, 161, 1626-1634.

F. Fregni Harvard Center for Non-Invasive Brain Stimulation, Beth Israel Deaconess Medical Center, Harvard Medical School, 330 Brookline Avenue, Boston, MA 02215, USA.

E-mail: ffregni@hsph.harvard.edn

P. S. Boggio Psychiatry Department, University of São Paulo, São Paulo, Brazil

M. Nitsche Department of Clinical

Neurophysiology, George-August-University, Göttingen, Germany

A. Pascual-Leone Harvard Center for NonInvasive Brain Stimulation, Beth Israel Deaconess Medical Center, Harvard Medical School, Boston, USA

\section{NICE recommendations for valproate treatment are unhelpful}

The National Institute for Clinical Excellence (NICE) guidelines for treatment of mania recommend that consideration be given to olanzapine and semisodium valproate as first-line treatments (NICE, 2003). They state that valproate can rarely cause severe liver damage and assert that liver function should be assessed before and during therapy, saying 'tests that reflect protein synthesis, particularly prothrombin time are most relevant'. They continue: 'Blood tests (blood cell count, including platelet count, bleeding time and coagulation tests) are therefore recommended'. Most readers will be familiar with the concept of the bleeding time only through the immortal lines delivered by James Robertson Justice in the film Doctor in the House (1954). It is a rarely indicated test of platelet function which requires making a $3 \mathrm{~cm}$ cut on a patient's forearm and timing how long it takes for the bleeding to stop. Clearly such a test would not be acceptable to a substantial proportion of patients with mania.

The recommendation seems a non sequitur. Saying that valproate can cause liver damage and that 'therefore' these investigations should be performed does not make sense because, with the exception of the coagulation tests, they are not indicators of hepatic function. In fact, the investigations are not recommended by the British National Formulary (BNF) but in the summary product characteristics for semisodium valproate (available at http:// emc.medicines .org.uk). It is here that it is stated that valproate can cause the frequent occurrence of thrombocytopenia, and it is here that the investigations listed are recommended.

It would strain credulity to believe that British doctors routinely measure bleeding time prior to initiating valproate therapy. Yet if a patient were to suffer ill effects, then having ignored recommendations found both in the summary product characteristics and in NICE guidelines could make an action for negligence difficult to defend.

Even setting aside the bleeding time, the advice to perform more straightforward investigations remains problematic. Faced with a manic patient, one is unlikely to feel enthusiastic about holding off treatment until a prothrombin time has been obtained. Instead, one will be tempted to choose an alternative treatment which can be started immediately, such as haloperidol. The BNF does not recommend that these blood tests be performed before starting valproate and there is no evidence base to show that carrying them out pre-treatment will produce a better outcome. The advice seems to have been included in the NICE guidelines in a thoughtless way, without regard to the possibility that unnecessary investigations will make a particular treatment option less acceptable to both doctors and patients. If recommendations about treatment are to be evidence-based, then so must be the recommendations about accompanying investigations.

National Institute for Clinical Excellence (2003)

Olanzapine and Valproate Semisodium in the Treatment of Acute Mania Associated with Bipolar I Disorder. Technology Appraisal 66. London: NICE.

D. Curtis, M. Kerr East London and City Mental Health Trust, Department of Adult Psychiatry, Royal London Hospital, Whitechapel, London EI IBB, UK. E-mail: david.curtis@qmul.ac.uk

\section{Combating editorial racism}

Peter Tyrer (2005) has set out a number of ways by which the British Journal of Psychiatry will attempt to minimise editorial racism and he acknowledges that this is only the beginning of a long journey. Nevertheless, he ought to be congratulated for his vision. His proposal to increase the number of corresponding editors from low- and middle-income countries is commendable, although I would like to see an 
increase in peer reviewers who have, like corresponding editors, an understanding of the issues in these countries. Otherwise, reviewers, who I am sure are fair-minded professionals, will continue to judge papers from poorer countries on the same basis as those submitted from rich countries, thereby perpetuating the problem of disproportionate publication. Surely there must be reviewers who will undertake this task - if not, appropriate professionals need to be encouraged to get involved so that they can make a significant contribution to ending editorial racism. Additionally, their participation will also encourage greater opportunities for publications from researchers from poorer countries which in itself, I believe, is a worthy cause.

Tyrer, P. (2005) Combating editorial racism in psychiatric publications. British Journal of Psychiatry, $\mathbf{1 8 6}$

S. Ramaiah Walsall NHS Primary Care Trust, Lichfield House, 27-3I Lichfield Street, Walsall WSI ITE, UK. E-mail: sam.ramaiah@walsall.nhs.uk

\section{Reading habits of British psychiatrists}

Jones et al's (2004) reassuring article that British psychiatrists read British journals may indicate the preoccupation of the British with British services. It would not be surprising to find that British people use the Royal Mail, watch the BBC or ITV, read British newspapers, fly British Airways (I wonder)! Tables 1 and 2, however, reveal another interesting observation, which the authors did not address in their otherwise interesting article. Advances in Psychiatric Treatment was more often read by those without academic commitments, in all the age groups. The difference in the adult psychiatric group is quite marked $17 \%$ of psychiatrists without academic commitments read Advances, compared with only $2 \%$ of those with academic commitments, a difference which may even be statistically significant. These trends are maintained in Table 2, where another difference between academic and nonacademic psychiatrists emerges: academic psychiatrists ranked the American Journal of Psychiatry, Archives of General Psychiatry, Biological Psychiatry and the Journal of Psychopharmacology higher than did psychiatrists without academic commitments. One could infer that psychiatrists without academic commitments preferred journals like Advances in Psychiatric Treatment, which have practical, management-related reviews and updates, and psychiatrists with academic commitments preferred research-based journals. Or these differences could confirm the Editor's hunch that Advances in Psychiatric Treatment will gradually become more popular (Tyrer, 2004).

Jones, T., Hanney, S., Buxton, B., et al (2004) What British psychiatrists read. Questionnaire survey of journal usage among clinicians. British Journal of Psychiatry, 185, 25I-257.

Tyrer, P. (2004) From the Editor's desk. British Journal of Psychiatry, 185, 276.

S. K. Chaturvedi National Institute of Mental Health \& Neurosciences, Bangalore 560029, India. E-mail: skchatur@yahoo.com

\section{One hundred years ago}

\section{'Kinds of insanity'}

THE preparation of a new set of statistical tables by the Medico-Psychological Association of Great Britain and Ireland for the annual recording of the vast clinical and pathological data and returns of all public asylums in the future has brought forward the inevitable question of the nomenclature and classification of the insanities. Dr. C. A. Mercier, in an article in the Journal of Mental Science for January, deals with the "kinds of insanity" which he thinks fulfil the necessary conditions of true diseases. The arrangement suggested is first to separate congenital from non-congenital cases of insanity. The congenital cases would include all idiots and most imbeciles. The classification of these is a matter of subordinate importance, whereas the division of the insanities proper into natural groups is the main desideratum in mental science and the most important aid to clearness of thought of diagnosis, and of prognosis. Cases of insanity are proposed to be considered in one of two classes viz., general paralysis (paralytic dementia) and non-paralytic insanity. Dr. Mercier suggests that the latter class contains "diseases sufficiently distinct that merit the same separation that is given to general paralysis.... As to general paralysis the symptoms are so distinct that it is recognisable at every stage in its progress. It has a definite history, runs a definite course, and forms a complete clinical picture separable from that of any other form of insanity." Examining other varieties of insanity and their titles or claims to be called diseases, Dr. Mercier would admit "acute delirious mania" owing to its characteristic symptoms and its course as a definite variety of insanity. "The clinical picture of acute delirious mania is distinct and prevents it from being confused with any other type of insanity. On the contrary," he says, "puerperal insanity presents us with no distinct clinical picture. The very fact that it has been divided into puerperal mania and puerperal melancholia is proof of what I say. Puerperal insanity is acute insanity occurring within an uncertain time of childbirth, and if the antecedent of childbirth is disregarded there is nothing whatever in the clinical picture of the disease that is different from other causes of acute insanity that have no connexion with the puerperium or even in acute insanity occurring in men." The insanity of pregnancy is regarded as having a much better right to be considered a disease, "for the fact of pregnancy is a continuing feature in the clinical picture, a feature which at once marks off the case from all other cases of insanity." What is true of the insanity of pregnancy, he adds, is emphatically true of the insanity of lactation. It is an insanity of exhaustion - of innutrition - and differs in no respect from other cases of insanity of similar origin. Few cases of insanity occurring at the menopause in women deserve recognition as a separate variety 